OPEN ACCESS

Edited by:

Hui Wu,

East China University of Science and Technology, China

Reviewed by: Feng Qi,

Fujian Normal University, China Lujing Ren,

Nanjing Tech University, China

*Correspondence:

Tatsuo Kurihara

kurihara@scl.kyoto-u.ac.jp

${ }^{\dagger}$ These authors share first authorship

Specialty section:

This article was submitted to Microbial Physiology and Metabolism,

a section of the journal

Frontiers in Microbiology

Received: 09 March 2020 Accepted: 04 May 2020

Published: 26 May 2020

Citation:

Ogawa T, Hirose K, Yusuf Y, Kawamoto $J$ and Kurihara T (2020) Bioconversion From

Docosahexaenoic Acid

to Eicosapentaenoic Acid in the Marine Bacterium Shewanella

livingstonensis Ac10

Front. Microbiol. 11:1104. doi: 10.3389/fmich.2020.01104

\section{Bioconversion From} Docosahexaenoic Acid to Eicosapentaenoic Acid in the Marine Bacterium Shewanella livingstonensis Ac10

\author{
Takuya Ogawa', Kazuki Hirose ${ }^{\dagger}$, Yustina Yusuft, Jun Kawamoto and Tatsuo Kurihara* \\ Molecular Microbial Science, Institute for Chemical Research, Kyoto University, Kyoto, Japan
}

Eicosapentaenoic acid (EPA) and docosahexaenoic acid (DHA), which belong to the same class of long chain $\omega-3$ polyunsaturated fatty acids (PUFAs), are present in marine $\gamma$-proteobacteria. In contrast to their de novo biosynthesis that has been intensively studied, their metabolic fates remain largely unknown. Detailed information regarding bacterial $\omega-3$ PUFA metabolism would be beneficial for understanding the physiological roles of EPA/DHA as well as the industrial production of EPA, DHA, and other PUFAs. Our previous studies revealed that the EPA-producing marine bacterium Shewanella livingstonensis Ac10 produces EPA from exogenous DHA independently of de novo EPA biosynthesis, indicating the presence of an unidentified metabolic pathway that converts DHA into EPA. In this study, we attempted to reveal the molecular basis for the bioconversion through both in vivo and in vitro analyses. Mutagenesis experiments showed that the gene disruption of $\mathrm{fadH}$, which encodes an auxiliary $\beta$-oxidation enzyme 2,4-dienoyl-CoA reductase, impaired EPA production under DHA-supplemented conditions, and the estimated conversion rate decreased by $86 \%$ compared to that of the parent strain. We also found that the recombinant FadH had reductase activity toward the 2,4-dienoyl-CoA derivative of DHA, whereas the intermediate did not undergo $\beta$-oxidation in the absence of the FadH protein. These results indicate that a typical $\beta$-oxidation pathway is responsible for the conversion. Furthermore, we assessed whether DHA can act as a substitute for EPA by using an EPA-less and conversion-deficient mutant. The cold-sensitive phenotype of the mutant, which is caused by the lack of EPA, was suppressed by supplementation with EPA, whereas the DHA-supplementation suppressed it to a lesser extent. Therefore, DHA can partly substitute for, but is not biologically equivalent to, EPA in S. livingstonensis Ac10.

Keywords: eicosapentaenoic acid, docosahexaenoic acid, marine bacteria, bioconversion, $\beta$-oxidation

Abbreviations: ACOX, acyl-CoA oxidase; CoA, coenzyme A; Cm, chloramphenicol; DHA, docosahexaenoic acid; EPA, eicosapentaenoic acid; ESI, electrospray ionization; FAME, fatty acid methyl ester; GC, gas chromatography; HEPES, $N$ 2-hydroxyethylpiperazine- $N^{\prime}$-2-ethanesulfonic acid; Km, kanamycin; MS, mass spectrometry; PL, phospholipid; PUFA, polyunsaturated fatty acid; Rf, rifampicin; Ura, uracil. 


\section{INTRODUCTION}

Various marine $\gamma$-proteobacteria produce $\omega-3$ long chain polyunsaturated fatty acids (PUFAs). Among them, a group of bacteria, which includes species Shewanella pneumatophori SCRC-2738 and Photobacterium profundum SS9, mainly produces (all- $Z$ ) eicosa-5,8,11,14,17-pentaenoic acid (EPA) (Yazawa, 1996; Nogi et al., 1998). Another group includes other species, such as Colwellia psychrerythraea H34 and Moritella marina MP-1, which mainly produce (all- $Z$ ) docosa4,7,10,13,16,19-hexaenoic acid (DHA) (DeLong and Yayanos, 1986; Hashimoto et al., 2015). As are found in and beneficial to a broad range of organisms, including mammals, nematodes, fungi, and algae, EPA and DHA are physiologically important for marine bacteria and have been proven to render the cells resistant to hostile environments, such as low temperatures and high hydrostatic pressures, and to hydrogen peroxide-induced oxidative stress (Nishida et al., 2006; Kawamoto et al., 2009, 2011). Bacterial PUFA production was previously believed to be limited to marine bacteria, but has since also been reported in recent years in members of $\delta$-proteobacteria retrieved from soil samples (Garcia et al., 2011, 2016).

The past two decades of research have uncovered a bacterial biosynthetic route for EPA/DHA that is unique in employing polyketide synthase-like multifunctional enzymes. The enzymes catalyze repeated cycles of decarboxylative condensation of malonyl-CoA with a growing fatty acyl group, which originates from the acetyl group, and $\mathrm{C}=\mathrm{C}$ bond-forming dehydration, and eventually release either EPA or DHA as the final product (Yoshida et al., 2016). The genes for PUFA synthesis are spread widely among the above-mentioned bacteria, but their gene products are composed of different catalytic domains and provide different final products, such as EPA, DHA, or other olefinic lipids (Orikasa et al., 2009; Shulse and Allen, 2011; Hayashi et al., 2019). The bacterial PUFA biosynthesis system differs from the eukaryotic system wherein PUFAs are produced from a pre-existing fatty acid, e.g., $\alpha$-linolenic acid [namely (all- $Z$ ) octadeca-9,12,15-trienoic acid], by the alternating actions of multiple elongases and desaturases (Metherel and Bazinet, 2019). In bacteria, EPA and DHA are generally introduced into the $s n-2$ position of glycerophospholipids and constitute the lipid bilayer of cellular membranes. The membrane-bound enzyme lysophosphatidic acid acyltransferase ( $\mathrm{PlsC}$ ) is responsible for the acylation step (Cho et al., 2012; Ogawa et al., 2018). However, in contrast to the biosynthetic pathways, the metabolic fates of EPA and DHA, such as degradation and conversion in bacteria, have not been well studied.

Shewanella livingstonensis Ac10 is a psychrotrophic bacterium originally isolated from Antarctic seawater and produces EPA, but not DHA, in response to low temperatures (Kawamoto et al., 2009). Like other EPA- and DHA-producing bacteria, S. livingstonensis Ac10 utilizes polyketide synthase-like enzymes to produce EPA de novo, and mutants that lack the genes coding for these enzymes produce no EPA, indicating that the polyketide synthase pathway is solely responsible for de novo EPA biosynthesis in this bacterium (Kawamoto et al., 2009). To investigate the physiological roles of EPA, we previously characterized the EPA-less mutant $(\triangle \mathrm{EPA})$ of $S$. livingstonensis Ac10. The mutant showed cold-sensitive phenotypes, such as growth retardation and aberrantly elongated cell shapes, when grown at $4^{\circ} \mathrm{C}$, while its phenotypes were similar to those of the wild-type when grown at $18^{\circ} \mathrm{C}$ (Kawamoto et al., 2009). The defects were suppressed by supplementation with a synthetic phospholipid (PL) that has EPA as its acyl chain (Sato et al., 2008; Kawamoto et al., 2009) and with free EPA (Tokunaga et al., 2017). Notably, a synthetic DHA-containing PL also suppressed the growth defects despite the absence of DHA in a wild-type cell of S. livingstonensis Ac10 (Sato et al., 2008). However, whether DHA (moiety) was beneficial to the bacterium remained ambiguous because PL analysis showed that the $\triangle \mathrm{EPA}$ cells unexpectedly produced EPA upon supplementation with DHA-containing PL. This finding indicated the presence of an unidentified metabolic route that generates EPA from exogenous DHA, thereby bypassing the loss of de novo EPA biosynthesis. Such a pathway has also been proposed for Escherichia coli (Watanabe et al., 1994), although the underlying mechanism is yet to be elucidated.

In this study, we attempted to reveal the conversion mechanism by both in vivo mutagenesis experiments and in vitro enzymatic assays and found that it is mediated through a typical $\beta$-oxidation pathway that involves an auxiliary $\beta$-oxidation enzyme 2,4-dienoyl-CoA reductase. Furthermore, as we obtained a mutant that no longer metabolizes DHA to EPA, we reassessed the biological equivalency of EPA and DHA by using an EPA-less and conversion-deficient mutant.

\section{MATERIALS AND METHODS}

\section{Reagents}

EPA and DHA were purchased from Nacalai Tesque (Kyoto, Japan), coenzyme A (CoA) from Oriental Yeast (Tokyo, Japan), and acyl-CoA oxidase (ACOX) from Asahi KASEI (Tokyo, Japan). All other chemicals used were of analytical grade.

\section{Bacterial Strain and Cultivation}

The $\triangle \mathrm{EPA}$ strain, which was derived from the $\triangle p y r F$ strain of $S$. livingstonensis Ac10 by deleting the orf5 gene (Ito et al., 2016), and its derivatives were cultivated at $4^{\circ} \mathrm{C}$ in Luria-Bertani (LB) medium that was, when indicated, supplemented with 50 $\mu \mathrm{g} / \mathrm{mL}$ kanamycin $(\mathrm{Km}), 50 \mu \mathrm{g} / \mathrm{mL}$ chloramphenicol $(\mathrm{Cm}), 50$ $\mu \mathrm{g} / \mathrm{mL}$ rifampicin (Rf), and/or $40 \mu \mathrm{g} / \mathrm{mL}$ uracil (Ura). EPA and DHA were dissolved in ethanol and added to LB medium at a final concentration of $32 \mu \mathrm{M}$ (or $128 \mu \mathrm{M}$ when indicated). The transformants of $E$. coli S17-1/ $\lambda$ pir were cultured in LB medium supplemented with $50 \mu \mathrm{g} / \mathrm{mL} \mathrm{Km}$ or $\mathrm{Cm}$, and those of E. coli BL21(DE3) were cultured in LB medium supplemented with 100 $\mu \mathrm{g} / \mathrm{mL}$ ampicillin.

Bacterial growth at $6^{\circ} \mathrm{C}$ in the presence and absence of 128 $\mu \mathrm{M}$ EPA or DHA was monitored by measuring the optical density at $600 \mathrm{~nm}\left(\mathrm{OD}_{600}\right)$ using a rocking incubator TVS062CA (ADVANTEC, Tokyo, Japan). The cell morphology at $\mathrm{OD}_{600}$ of about 1.0 was observed using a BX40F4 trinocular microscope (OLYMPUS, Tokyo, Japan) equipped with a Moticam 2500 digital 
microscope (Shimadzu Rika, Tokyo, Japan). The software ImageJ version $1.52 \mathrm{q}^{1}$ was used to quantify the longitudinal cell length.

\section{Mutagenesis}

Single-crossover homologous recombination-based mutagenesis was carried out to disrupt six putative $f a d H$ genes, i.e., sl_221, sl_453, sl_1274, sl_1301, sl_1351, and sl_1692 (Accession numbers: LC528929, LC528930, LC528931, LC528932, LC528933, and LC528934, respectively). Approximately $500 \mathrm{bp}$ internal regions of the genes were amplified by PCR using Q5 DNA polymerase (New England Biolabs, Ipswich, MA, United States), the genome of S. livingstonensis Ac10 as the template, and the primer pairs listed in Table 1. The linearized

${ }^{1}$ https://imagej.nih.gov/ij/
pKNOCK-Km was prepared by inverse PCR using the primer pairs listed in Table 1. Each of the amplified gene fragments was inserted into the SmaI site of pKNOCK-Km using a NEBuilder HiFi DNA Assembly kit (New England Biolabs), following the manufacturer's instructions. The competent cells of E. coli S17-1/ $\lambda$ pir were transformed with the resulting plasmids, which were thereafter screened on a Km-supplemented LB agar plate. After the transformants and $\triangle \mathrm{EPA}$ strain of $S$. livingstonensis Ac10 were cultivated in $\mathrm{LB}$ medium to $\mathrm{OD}_{600}$ of about 1.0, 100 $\mu \mathrm{L}$ of each culture was mixed and spread onto an LB agar plate. The bacterial lawns that formed after overnight incubation at $18^{\circ} \mathrm{C}$ were collected and suspended in $1 \mathrm{~mL}$ of $\mathrm{LB}$ medium, and $200 \mu \mathrm{L}$ of the cell suspension was spread onto the LB agar plate supplemented with $\mathrm{Km}$ and Rf. The single colonies that formed on the selection plate were screened by colony PCR

TABLE 1 | Primers used in this study.

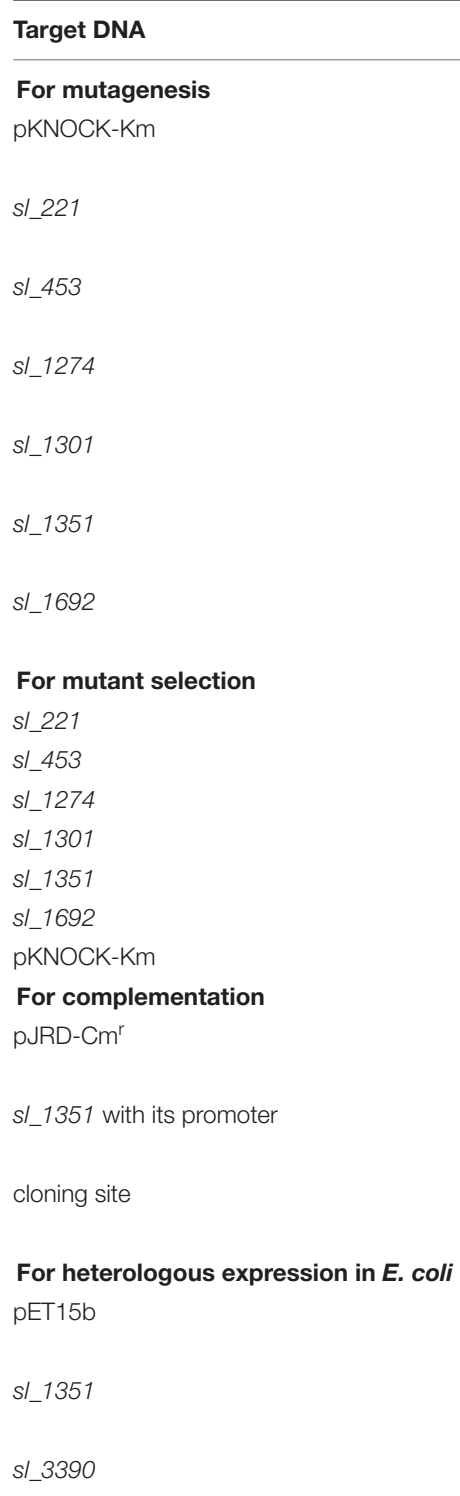

DNA sequences of forward (Fw) and reverse (Rv) primers

FW, 5'-GGGCTGCAGGAATTCGATATCAAGC-3'

Rv, 5'-GGGGGATCCACTAGTTCTAGAGCG-3'

Fw, 5'-CTAGAACTAGTGGATCCCCCCTACCCAAATACCCCTGGTTTATTACTCC-3'

Rv, 5' -ATATCGAATTCCTGCAGCCCACTCAGGTAACAAGTAGTCAAACACGTCAC-3'

FW, 5' -CTAGAACTAGTGGATCCCCCATCATTACCATAATTTAGCCCAGTCTGGTG-3'

Rv, 5' -ATATCGAATTCCTGCAGCCCTTACTGACTATTTCGAGCAATATTCTGGCG-3'

Fw, 5'-CTAGAACTAGTGGATCCCCCGAAGTTGCAGCATATTACCGCAGAC-3'

Rv, 5' -ATATCGAATTCCTGCAGCCCACTATTTCAACACCAAAGCGAGTACGATT-3'

FW, 5'-CTAGAACTAGTGGATCCCCCTGAATATTATGCTCAACGCGCTTCAGC-3'

Rv, 5'-ATATCGAATTCCTGCAGCCCACCACCATATTCGTCAATACGATTATTCG-3'

Fw, 5'-CTAGAACTAGTGGATCCCCCAAGCTITCTTGGCAGGTGGGTA-3'

Rv, 5'-ATATCGAATTCCTGCAGCCCACCGGTATTAATAATACTTACGGCTGCTTG-3'

FW, 5' -CTAGAACTAGTGGATCCCCCACCAGACGATGCATTGTTCAACTTG-3'

Rv, 5'-ATATCGAATTCCTGCAGCCCATCGGCACCACATTTAAGTCTTACTTGC-3'

FW, 5'-ACATTCTTGTACGATTAGCGCTAAATAAGC-3'

Fw, 5'-TATGCTGGTTAGGTTCAGTATGTTGCGTG-3'

Fw, 5'-TCTGTTAATGGCGTCATCCCAGGC-3'

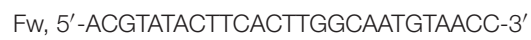

FW, 5'

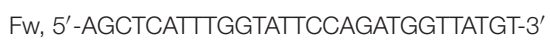

Rv, 5'-ACGTGTTCCGCTTCCTTTAGCA-3'

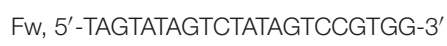

Rv, 5'-CGTAATCCATGGATCAAGAG-3'

FW, 5' -GATCCATGGATTACGACGTTGATGATGAACTTACGGATACC-3'

Rv, 5'-CTATAGACTATACTATTAAATACTCATCGCAAGTTCTGCACC-3'

FW, 5'-CCAGCTCTTTCTGCAGTTCATTC-3'

Rv, 5'-CTGGATTTCACTGATGAGAATATCGTCG-3'

FW, 5'-ATGGCTGCCGCGCGGCAC-3'

Rv, 5'-GGATCCGGCTGCTAACAAAG-3'

Fw, 5' -GTGCCGCGCGGCAGCCATATGTCGTTTCCACACTTATTAGAACCT-3'

Rv, 5'-CTTGTTAGCAGCCGGATCCTTAAATACTCATCGCAAGTTCTGCACC-3'

Fw, 5'-GTGCCGCGCGGCAGCCATATGATCTACCAAAGCCCTACCATTCAG-3'

Rv, 5' -CTTGTTAGCAGCCGGATCCTTAGGCTTGGTAGTAACTACCATTGTTGG-3' 
using the diagnostic primers listed in Table $\mathbf{1}$ to obtain six fadH-disrupted mutants, which are hereafter designated as, for example, $\Delta \mathrm{EPA} / s l \_1351$.

For complementation, the $s l \_1351$ gene together with its 249 bp-upstream region (Accession number: LC528933), which contains a putative promoter predicted on the Neural Network Promoter Prediction website ${ }^{2}$, and pJRD-Cm ${ }^{\mathrm{r}}$ (Toyotake et al., 2018) were PCR-amplified using the primer pairs listed in Table 1. The two PCR products were fused using an InFusion cloning kit (Takara Bio, Shiga, Japan) to obtain pJRDsl_1351. E. coli S17-1/ $\lambda$ pir transformed with the plasmid and the $\Delta \mathrm{EPA} / s_{-} 1351$ strain were cultivated in $\mathrm{LB}$ medium to $\mathrm{OD}_{600}$ of 0.2-0.3, co-cultured on an LB agar plate, then screened on an LB agar plate supplemented with $\mathrm{Cm}$ and $\mathrm{Km}$. The mutant harboring pJRD-sl_1351 was selected by colony PCR using the diagnostic primers listed in Table 1.

\section{Electrospray lonization (ESI)-Mass Spectrometry (MS) Analysis of PL Composition}

The parent and mutant strains were cultivated in $5 \mathrm{~mL}$ of Ura-supplemented LB medium at $4^{\circ} \mathrm{C}$. After the cells were grown to $\mathrm{OD}_{600}$ of about 1.0, they were harvested and subjected to Bligh and Dyer lipid extraction (Bligh and Dyer, 1959). The resulting lower (chloroform-methanol) phase was collected and dried under a stream of nitrogen gas, and the residual lipids were dissolved in methanol/acetonitrile (1:1) containing $0.1 \%$ triethylamine for ESI-MS analysis. The API3000 triple-quadrupole mass spectrometer (SCIEX, Ontario, Canada) equipped with an electrospray ion source was used to scan the total ions with the following parameters: polarity, negative; nebulizer gas, 8; curtain gas, 8 ; ion spray voltage, $-4,200 \mathrm{~V}$; declustering potential, $-30 \mathrm{~V}$; focusing potential, $-200 \mathrm{~V}$; and entrance potential, $-10 \mathrm{~V}$. In addition, EPA- and DHAcontaining PLs were selectively scanned in the precursor ion mode with target $\mathrm{m} / \mathrm{z}$ of 301.4 and 327.4, respectively, with the following parameters; collision energy, $-45 \mathrm{eV}$, and collision cell exit potential, $-15 \mathrm{~V}$.

\section{Gas Chromatography (GC)-Coupled MS Analysis of Fatty Acid Composition}

Fatty acid methyl esters (FAMEs) were prepared from the lipids extracted as described above following the methods reported previously (Ichihara and Fukubayashi, 2010). Briefly, the dried lipid extracts were dissolved in $0.2 \mathrm{~mL}$ of toluene, mixed with $1.5 \mathrm{~mL}$ of methanol and $0.3 \mathrm{~mL}$ of $8 \% \mathrm{HCl} / \mathrm{methanol}$, and incubated at $45^{\circ} \mathrm{C}$ for $14 \mathrm{~h}$. After cooling to ambient temperature, $1 \mathrm{~mL}$ of water and $n$-hexane, respectively, was added and the solution was vigorously shaken to extract FAMEs, followed by concentration to about $400 \mu \mathrm{L}$ under a stream of nitrogen gas. The FAMEs were analyzed using a Clarus SQ 8C mass spectrometer interfaced with a Clarus 680 gas chromatograph (Perkin Elmer, Wellesley, MA, United States) equipped with a DB- 1 capillary column $(30 \mathrm{~m} \times 0.25 \mathrm{~mm}, 0.25 \mu \mathrm{m}$; Agilent

${ }^{2}$ https://www.fruitfly.org/seq_tools/promoter.html
Technology, Santa Clara, CA, United States). The experiments were performed in triplicate, and the peak area for each FAME was divided by the sum of the peak areas for total FAMEs to calculate the fatty acid composition.

To estimate the conversion rate from DHA to EPA, $1 \mathrm{~mL}$ of bacterial culture was extracted via the Bligh-Dyer method, and the chloroform-methanol phase was dried, methyl-esterified, and analyzed by GC/MS, as described above. The experiments were performed in triplicate, and the peak area for EPA methyl ester was divided by the sum of the peak area for DHA and EPA methyl esters to calculate the conversion rate.

\section{Heterologous Expression and Purification of SI_1351 and SI_3390}

The genes $s l \_1351$ and $s l \_3390$ (Accession numbers: LC528933 and LC528935, respectively) were PCR-amplified using the genome of S. livingstonensis Ac10 as the template and the primer pairs listed in Table 1, while the linearized pET15b was prepared by inverse PCR using the primer pair listed in Table 1. The respective genes were inserted into the NdeI-BamHI site of pET15b using a NEBuilder HiFi DNA Assembly kit to obtain pET15b-sl_1351 and pET15b-sl_3390. E. coli BL21(DE3) transformed with pET15b-sl_1351 was cultured in $350 \mathrm{~mL}$ of ampicillin-supplemented $\mathrm{LB}$ medium at $37^{\circ} \mathrm{C}$ until $\mathrm{OD}_{600}$ reached 0.4 , and was subsequently cultured at $18^{\circ} \mathrm{C}$ for a further 22 h. E. coli BL21(DE3) transformed with pET15b-sl_3390 was cultured in the same way, except the protein expression was induced with $0.5 \mathrm{mM}$ isopropyl- $\beta$-D-1-thiogalactoside following the temperature down-shift. The cells were harvested by centrifugation and stored at $-30^{\circ} \mathrm{C}$ for future use.

The collected cells (approximately $1 \mathrm{~g}$ of wet cells) of E. coli BL21(DE3) harboring pET15b-sl_1351 were suspended in $5 \mathrm{~mL}$ of binding buffer $(20 \mathrm{mM}$ sodium phosphate, $\mathrm{pH} 7.4$, $500 \mathrm{mM} \mathrm{NaCl}, 20 \mathrm{mM}$ imidazole, and $0.1 \mathrm{mM}$ dithiothreitol) and disrupted by sonication. After the lysate was clarified by centrifugation $\left(20,000 \mathrm{~g}\right.$ for $30 \mathrm{~min}$ at $\left.4^{\circ} \mathrm{C}\right)$, the supernatant was recovered and loaded to a HisTrap FF $1 \mathrm{ml}$ column (GE healthcare, Chicago, IL, United States) pre-equilibrated with the binding buffer. The column was washed with $10 \mathrm{~mL}$ of wash buffer (20 mM sodium phosphate, pH 7.4, $500 \mathrm{mM} \mathrm{NaCl}$, $60 \mathrm{mM}$ imidazole, and $0.1 \mathrm{mM}$ dithiothreitol) then eluted with $5 \times 1 \mathrm{~mL}$ of elution buffer $(20 \mathrm{mM}$ sodium phosphate, $\mathrm{pH} 7.4$, $500 \mathrm{mM} \mathrm{NaCl}, 500 \mathrm{mM}$ imidazole, and $0.1 \mathrm{mM}$ dithiothreitol). The eluates were pooled and subjected to ultrafiltration with an Amicon Ultra $50 \mathrm{kDa}$ Cutoff Filter Unit (Merck, Darmstadt, Germany) to concentrate the recombinant proteins and dilute imidazole by repeated dilution-concentration. The protein profile was analyzed by SDS-PAGE (9\% acrylamide), and protein content was measured via the Bradford method. Recombinant Sl_3390 was similarly purified, except buffers containing no dithiothreitol were used.

\section{In vitro Enzymatic Assays}

Fatty acyl-CoAs were synthesized through the mixed-anhydride method, as described elsewhere (Ogawa et al., 2018). In the product analysis for $\mathrm{Sl} \_1351$, the $100 \mu \mathrm{L}$ reaction mixture 
containing $0.1 \mathrm{mM}$ DHA-CoA, 0.1 unit of ACOX, $1 \mu \mathrm{M}$ purified Sl_1351, $1 \mu \mathrm{M}$ purified Sl_3390, $0.2 \mathrm{mM} \mathrm{NADPH}, 1.0 \mathrm{mM}$ $N$-2-hydroxyethylpiperazine- $N^{\prime}$-2-ethanesulfonic acid (HEPES)$\mathrm{NaOH}$ ( $\mathrm{pH} 7.0$ ), and $0.12 \mathrm{mM}$ dithiothreitol was incubated at $20^{\circ} \mathrm{C}$ for $10 \mathrm{~min}$. The reaction mixture was placed on ice to terminate the reaction and then desalted with an Oasis HLB 1 cc cartridge (Waters, Milford, MA, United States; Magnes et al., 2005). The cartridge was washed with $3 \mathrm{~mL}$ of acetonitrile and equilibrated with $2 \mathrm{~mL}$ of $1.0 \mathrm{mM}$ HEPES-NaOH ( $\mathrm{pH} 7.0$ ), followed by sample loading. The cartridge was washed with $4 \mathrm{~mL}$ of water and finally eluted with $0.5 \mathrm{~mL}$ of acetonitrile/water (1:1) containing $15 \mathrm{mM}$ ammonium hydroxide. The eluates were analyzed by ESI-MS with the following parameters: polarity, positive; nebulizer gas, 8; curtain gas, 8; ion spray voltage, 4,200 V; declustering potential, $65 \mathrm{~V}$; focusing potential, $200 \mathrm{~V}$; and entrance potential, $10 \mathrm{~V}$.

In the product analysis for Sl_3390, the $100 \mu \mathrm{L}$ reaction mixture containing $0.1 \mathrm{mM}$ palmitoyl-CoA, 0.1 unit of ACOX, 1 $\mu \mathrm{M}$ purified Sl_3390, $0.2 \mathrm{mM}$ NAD, and $1.0 \mathrm{mM}$ HEPES-NaOH $(\mathrm{pH} 7.0)$ was incubated at $20^{\circ} \mathrm{C}$ for $10 \mathrm{~min}$. The product was desalted and subjected to ESI-MS analysis, as described above.

\section{RESULTS}

\section{The Conversion of Exogenous DHA Into EPA in the $\triangle E P A$ Strain}

In our previous study, the $\triangle \mathrm{EPA}$ strain was supplemented with DHA as a form of the acyl group of a synthetic PL (Sato et al., 2008). As S. livingstonensis Ac10 can incorporate exogenous free EPA and utilize it to form membrane PLs (Tokunaga et al., 2017), we supplemented the $\triangle \mathrm{EPA}$ strain with a free DHA in this study and analyzed its PL profiles by ESI-MS (Figure 1 and Supplementary Figure S1). In addition to a total ion scan, a precursor ion scan for ions of eicosapentaenoate $(\mathrm{m} / z$ 301.4) and docosahexaenoate $(\mathrm{m} / z$ 327.4) was performed to selectively detect EPA- and DHAcontaining PLs. Without supplementation of DHA, the parent strain produced EPA-containing, but not DHA-containing, PLs (Supplementary Figure S1A and Table 2), whereas neither were detected in $\triangle E P A$ cells (Supplementary Figure S1B). On the other hand, when grown in the presence of DHA, the parent strain produced both EPA- and DHA-containing PLs (Figure 1A and Table 2), indicating that the bacterium utilized exogenous DHA to form membrane PLs. It was also noted that the $\triangle$ EPA strain produced EPA- as well as DHA-containing PLs in DHA-supplemented conditions (Figure 1B and Table 2). These data suggested that S. livingstonensis Ac10 could produce EPA from exogenous DHA.

To confirm the occurrence of EPA in the $\triangle$ EPA cells, fatty acyl moieties of the extracted PLs were methyl-esterified and subjected to GC/MS analysis. As a result, EPA and DHA methyl esters, which eluted at $28.5 \mathrm{~min}$ and $33.2 \mathrm{~min}$, respectively (Figure 2A), were detected in the FAMEs of the DHAsupplemented cells (Figure 2B) and accounted for $6.0 \pm 0.3 \%$ and $1.8 \pm 0.1 \%$ of the total FAMEs, respectively. Therefore, EPA production in the DHA-supplemented $\triangle \mathrm{EPA}$ cells was unambiguously demonstrated and strongly supports the presence of a metabolic pathway that converts DHA into EPA. To estimate the rate of conversion from DHA to EPA, the bacterial culture was subjected to FAME analysis. The peak area for EPA methyl ester (corresponding to EPA produced) was divided by the sum of the peak area for EPA and DHA methyl esters (corresponding to DHA added), resulting in the conversion rate of $89.2 \pm 0.6 \%$.

\section{Mutagenesis Analysis of the Conversion Mechanism}

As two carbon units and one double bond are eliminated when EPA is formed from DHA, we presumed that the conversion is mediated through the $\beta$-oxidation pathway or a similar metabolic route. As depicted in Figure 3A, the typical bacterial $\beta$-oxidation reactions for saturated fatty acids are achieved by the actions of three proteins with four enzymatic activities, acyl-CoA dehydrogenase (FadE), 2-enoyl-CoA hydratase/3-hydroxyacylCoA dehydrogenase (FadB), and 3-ketoacyl-CoA thiolase (FadA) (DiRusso et al., 1999). Unsaturated fatty acids with a double bond at odd-numbered carbon atoms are likewise degraded. On the other hand, the auxiliary enzyme 2,4-dienoyl-CoA reductase $(\mathrm{FadH})$ is also required to metabolize unsaturated fatty acids, such as DHA, that have a double bond at even-numbered carbon atoms (You et al., 1989). Therefore, to investigate the conversion mechanism in S. livingstonensis Ac10, we examined the possible involvement of $\mathrm{fadH}$ genes by mutagenesis.

A homology search utilizing the amino acid sequence of a well-studied FadH from E. coli (Dommes and Kunau, 1984; Liang et al., 2000; Hubbard et al., 2003; Tu et al., 2008) as a query revealed that $S$. livingstonensis Ac10 has six FadH homologs. The homolog Sl_1351 has an N-terminal catalytic domain and C-terminal $\mathrm{NAD}(\mathrm{P}) \mathrm{H}$-binding domain as with E. coli FadH, whereas the other five homologs (S1_221, Sl_453, Sl_1274, Sl_1301, and Sl_1692) are composed of the catalytic domain only (Figure 3B). We disrupted each of the six $\mathrm{fadH}$ genes of the $\triangle \mathrm{EPA}$ strain, which were confirmed by the formation of a PCR product in diagnostic PCR (data not shown), and analyzed the PL profiles of the mutants grown in the presence of DHA. ESI-MS analysis of the extracted lipids showed that EPA-containing PL levels markedly decreased in the

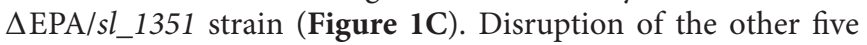
genes had no significant impact on EPA-containing PL levels (Supplementary Figure S2). GC/MS analysis of FAMEs also showed a decrease in EPA levels in the $\triangle \mathrm{EPA} /$ sl_$_{-} 1351$ strain (Figure 2C) and the conversion rate dropped to $12.3 \pm 0.9 \%$, which was 7.4 -fold lower than that of the $\triangle \mathrm{EPA}$ strain. For gene complementation, we introduced a plasmid (i.e., pJRDsl_1351) that carries $s l \_1351$ gene into the $\Delta \mathrm{EPA} / s l \_1351$ mutant, which was confirmed through diagnostic PCR (data not shown). The conversion ability was restored to a level comparable to that of the $\triangle$ EPA strain by complementation with pJRD-sl_1351 (Figures 1D, 2D) but not with an empty vector (Figures 1E, 2E). In addition, an unknown compound that eluted at $33.5 \mathrm{~min}$, close to the time at which DHA methyl ester eluted, was present in the FAMEs of the conversion-deficient cells (Figures 2C,E, asterisk) and showed a mass spectrum almost identical to that 

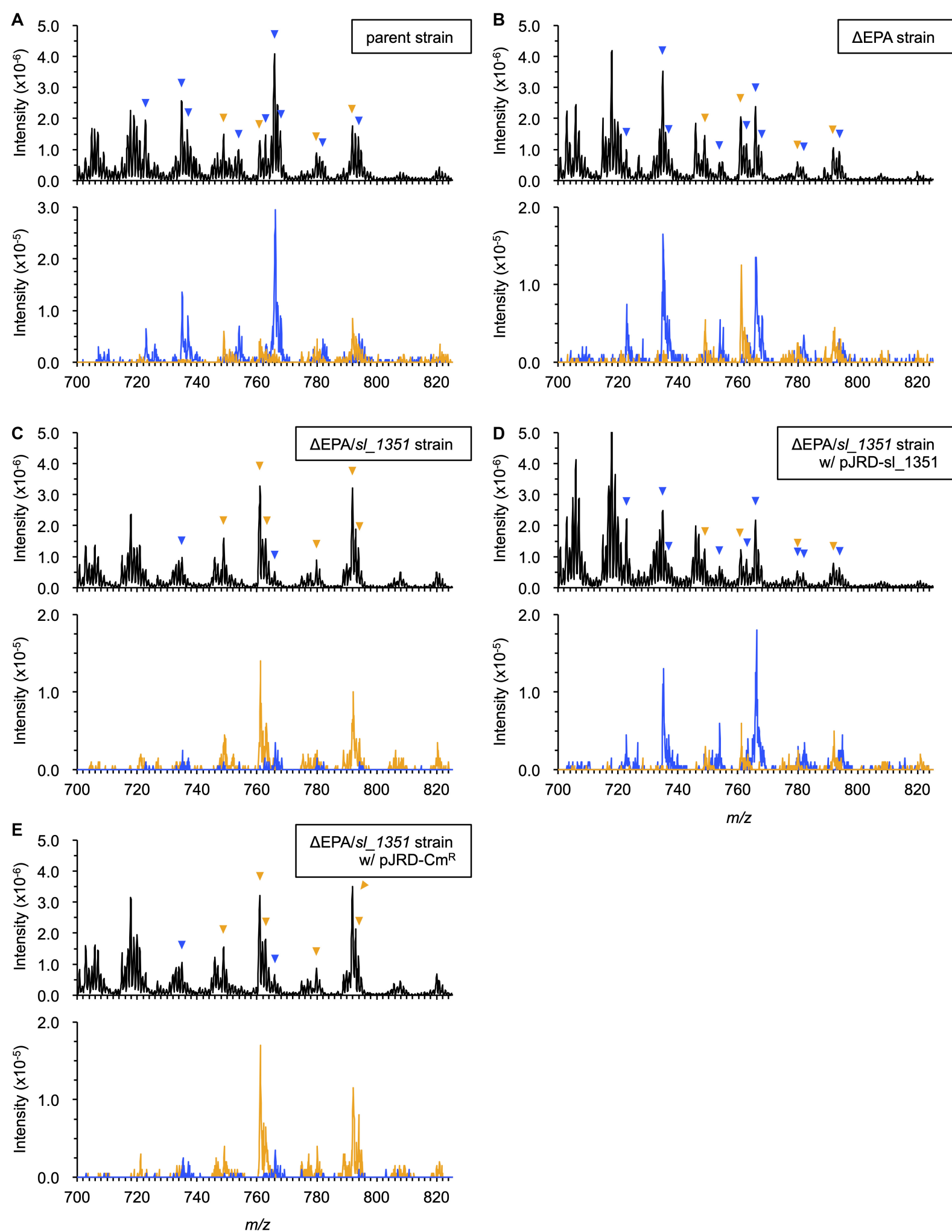

FIGURE 1 | ESI-MS analyses of PL compositions. The PLs were extracted from the parent strain (A), $\Delta$ EPA strain (B), $\Delta$ EPA/sI_1351 strain (C), $\Delta$ EPA/s/_1351 strain harboring pJRD-sI_1351 (D), and $\Delta$ EPA/sI_1351 strain harboring pJRD-Cm ${ }^{r}$ (E) grown in the presence of DHA. Three spectra of total ions (upper panel) and precursor ions for $\mathrm{m} / \mathrm{z}$ of 301.4 and 327.4 (blue and orange traces on lower panel, respectively) are shown. The arrowheads indicate EPA- (blue) and DHA-containing PL ions (orange), of which predicted fatty acyl compositions are shown in Table 2. 
of DHA methyl ester, with slight differences (data not shown). The peak probably represents the 2,4-dienoyl-CoA derivative of DHA (docosa-2,4,7,10,13,16,19-heptaenoyl-CoA), which can accumulate due to deficiency in the conversion. Although a small

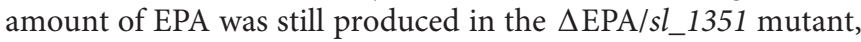
these results strongly suggested that S1_1351 plays a key role in DHA conversion.

\section{In vitro Assay of the Recombinant SI_1351 Protein}

To further examine the role of Sl_1351 in DHA conversion, the recombinant S1_1351 protein was heterologously produced in E. coli BL21(DE3) and purified by affinity chromatography (Figure 4A). The recombinant Sl_3390 protein, a putative FadB of $S$. livingstonensis Ac10, was also prepared in the same way and was found to have 2-enoyl-CoA hydratase, but not 3-hydroxyacyl-CoA dehydrogenase, activity (Supplementary Figure S3), most likely due to product inhibition in the absence of FadA (Binstock and Schulz, 1981). For the 2,4-dienoylCoA reductase assay, the substrate docosa-2,4,7,10,13,16,19heptaenoyl-CoA was prepared from DHA-CoA by using ACOX, and the production of docosa-2,7,10,13,16,19-hexaenoyl-CoA was validated by using the recombinant S1_3390 (Figure 4B).
DHA-CoA was incubated with the three proteins ACOX, Sl_1351, and Sl_3390, and the products were analyzed by ESI-MS.

Without the addition of the enzymes, DHA-CoA was detected primarily as an $[\mathrm{M}+\mathrm{H}]^{+}$ion with an $m / z$ of 1078.4 (Figure 4C).

TABLE 2 | Representative $m / z$ values of $[\mathrm{M}-\mathrm{H}]^{-}$ions of EPA- and DHA-containing PLs detected in ESI-MS analysis.

\begin{tabular}{lccc}
\hline & & \multicolumn{2}{c}{ Headgroup } \\
\cline { 3 - 4 } & & Ethanolamine & Glycerol \\
\hline Acyl chains & $15: 0 / 20: 5$ & 723.0 & 753.8 \\
& $16: 1 / 20: 5$ & 735.1 & 766.0 \\
& $16: 0 / 20: 5$ & 736.9 & 768.0 \\
$17: 1 / 20: 5$ & 748.9 & 780.2 \\
$17: 0 / 20: 5$ & 750.8 & 782.1 \\
& $18: 1 / 20: 5$ & 762.9 & 793.9 \\
$15: 0 / 22: 6$ & 749.1 & 779.9 \\
$16: 1 / 22: 6$ & 761.0 & 792.1 \\
$16: 0 / 22: 6$ & 763.0 & 793.9 \\
\hline
\end{tabular}

Fatty acyl chains are abbreviated as follows: 15:0, pentadecanoyl; 16:1, hexadecenoyl; 16:0, hexadecanoyl; 17:1, cyclopropane-containing hexadecanoyl; 17:0, heptadecanoyl; 18:1, octadecenoyl; 20:5, eicosapentaenoyl; 22:6, docosahexaenoyl.
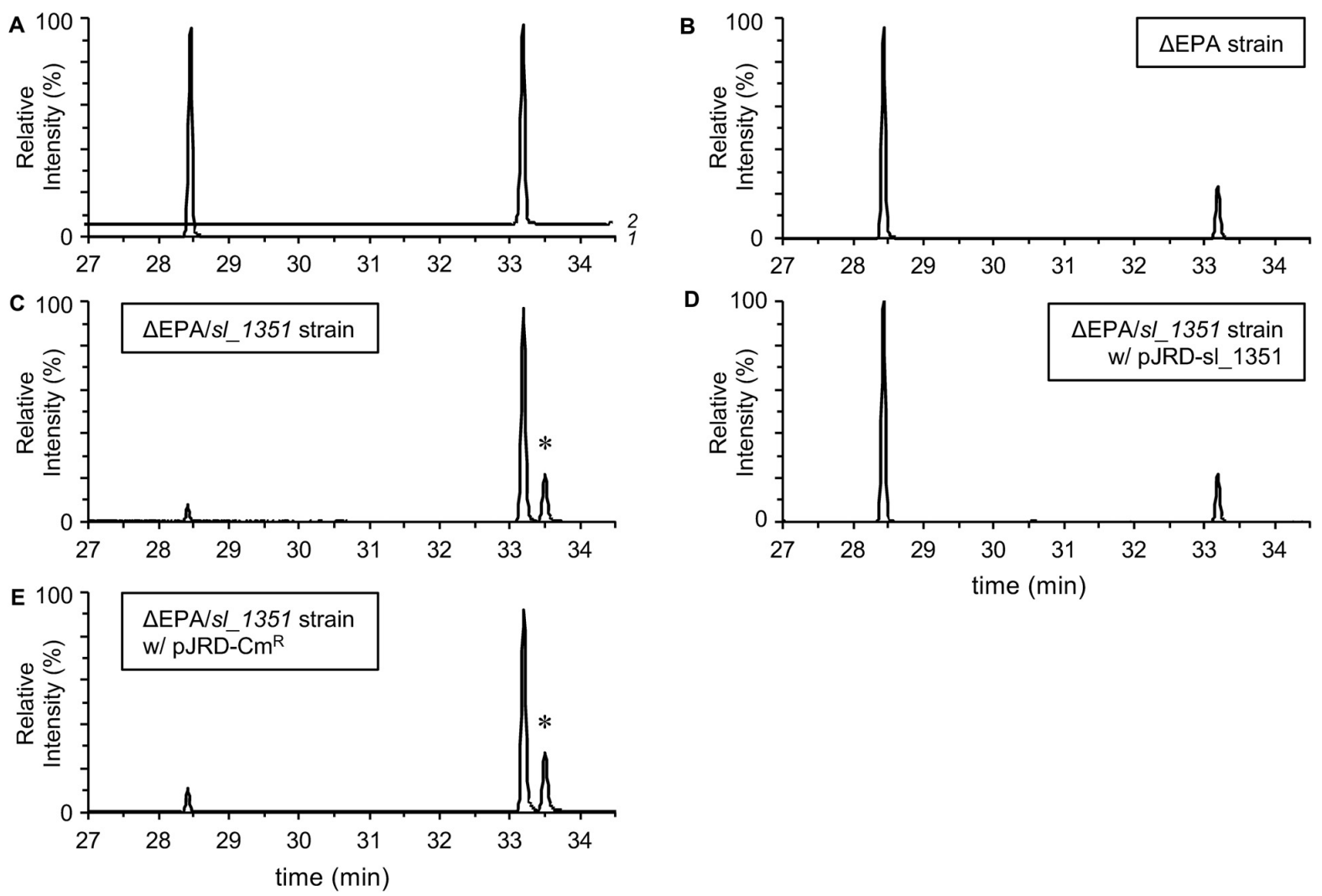

FIGURE 2 | GC/MS analyses of fatty acid compositions. Authentic EPA and DHA methyl esters (A; traces 1 and 2, respectively) and the FAMEs of the $\triangle$ EPA strain (B), $\Delta$ EPA/sl_1351 strain (C), $\Delta$ EPA/sI_1351 strain harboring pJRD-sl_1351 (D), and $\Delta$ EPA/sl_1351 strain harboring pJRD-Cm ${ }^{r}$ (E) grown in the presence of DHA were analyzed. An asterisk indicates the peak putatively representing methyl docosaheptaenoate. 


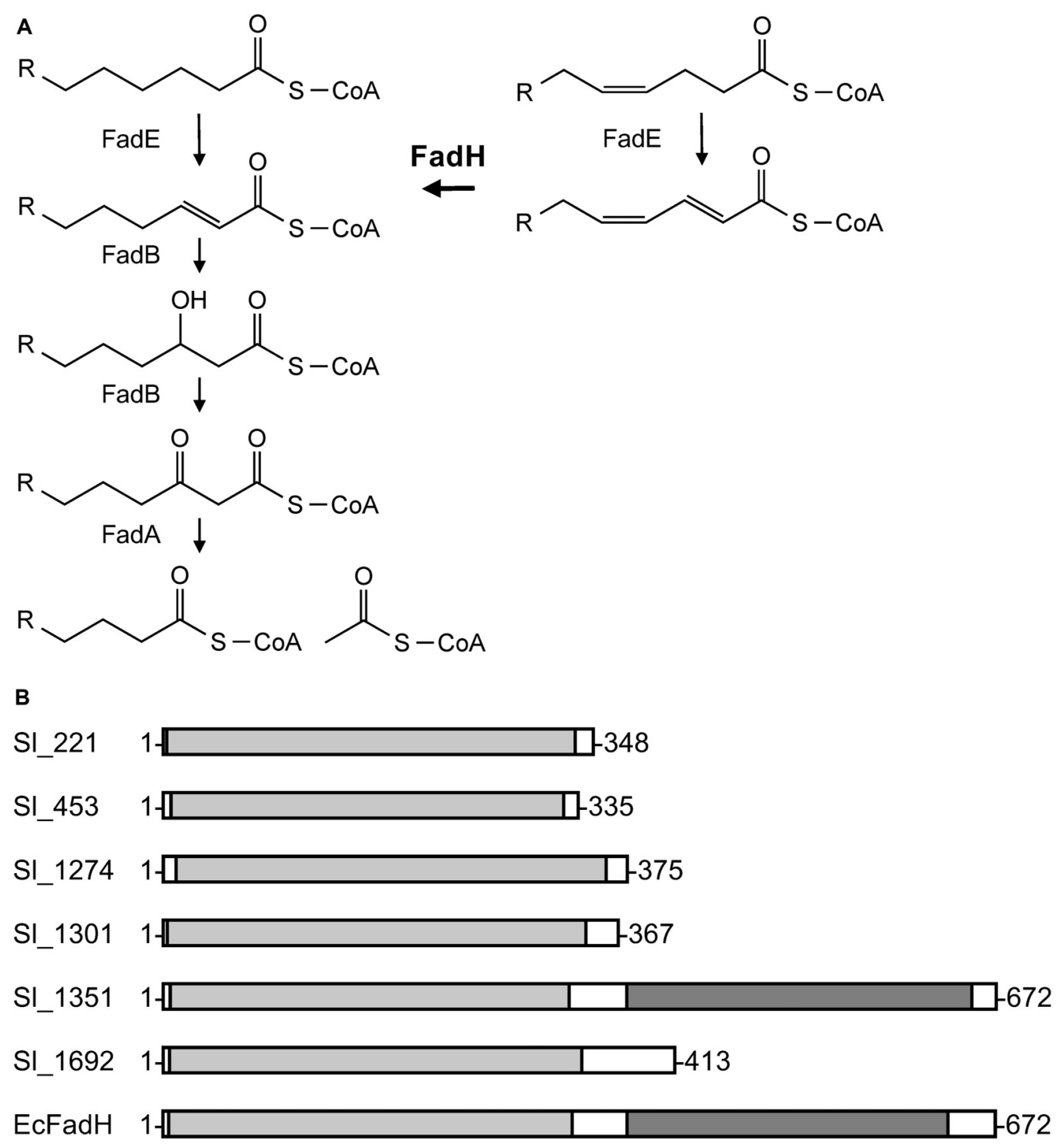

FIGURE 3 | General $\beta$-oxidation reactions (A) and the domain structures of the six FadH homologs (B). (A) FadE, acyl-CoA dehydrogenase; FadB, enoyl-CoA hydratase/3-hydroxyacyl-CoA dehydrogenase; FadA, 3-ketoacyl-CoA thiolase; and FadH, 2,4-dienoyl-CoA reductase. (B) The image was generated on the MOTIF Search website (https://www.genome.jp/tools/motif/) and modified. The light gray represents a catalytic domain and the dark gray NAD(P)H-binding domain. EcFadH represents FadH from E. coli.

This value was in good agreement with the theoretical monoisotopic mass of DHA-CoA, 1078.3522. The addition of ACOX decreased the $m / z$ value by 2 (Figure 4D), indicating the removal of two hydrogen atoms to form docosaheptaenoyl-CoA (Figure 4B, step 1). As expected, the addition of both ACOX and Sl_3390 did not cause hydration of the product (Figure 4E). When DHA-CoA was incubated with both ACOX and Sl_1351, an ion with an $m / z$ of 1078.4 was detected (Figure 4F), which likely represents the reduced product, docosa-2,7,10,13,16,19hexaenoyl-CoA (Figure 4B, step 2). The $m / z$ value of this ion is indistinguishable from the $[\mathrm{M}+\mathrm{H}]^{+}$ion of DHA-CoA (Figure 4C). However, the product served as the substrate of S1_3390 and was hydrated with the $\mathrm{m} / \mathrm{z}$ value increasing by 18 in the presence of all the three enzymes (Figure 4B, step 3 and
Figure 4G), whereas DHA-CoA was not directly hydrated by Sl_3390 (Figure 4H). Therefore, the product of Sl_1351 would be docosa-2,7,10,13,16,19-hexaenoyl-CoA, a typical $\beta$-oxidation intermediate. Altogether, these data suggested that $s l$ 1351 codes for 2,4-dienoyl-CoA reductase required to metabolize DHA and support the results of the mutagenesis experiments.

\section{The Effects of DHA Supplementation on the Cold-Sensitive Phenotypes of the $\triangle$ EPA Strain}

EPA and DHA are long carbon chain compounds (20 and 22 carbons, respectively) that are multiply unsaturated (five and six double bonds, respectively) and categorized into the same 


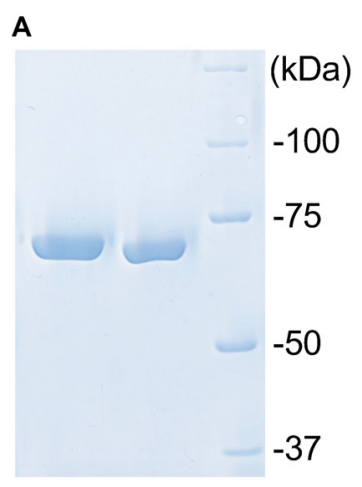

B<smiles>[B]C/C=C/CCC(=O)SCC</smiles>

(M)

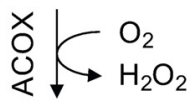<smiles>[R]CC=CC=CC(=O)SOC(=O)O</smiles>

(M-2)

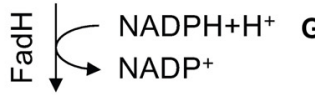<smiles>[R]CCC/C=C/C(=O)SO</smiles>

(M)<smiles>[R]CCCC(O)CC(=O)SCC(C)(C)CO</smiles>

C

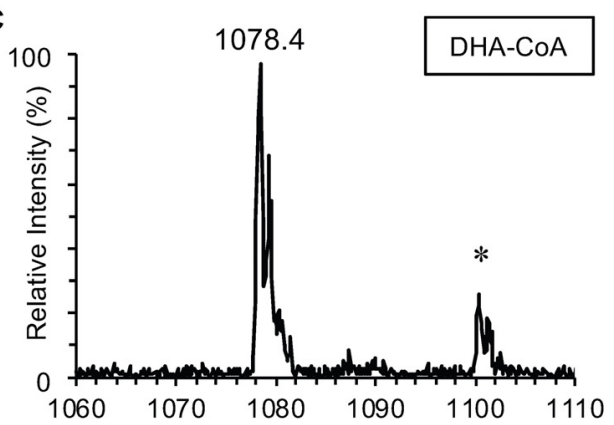

D

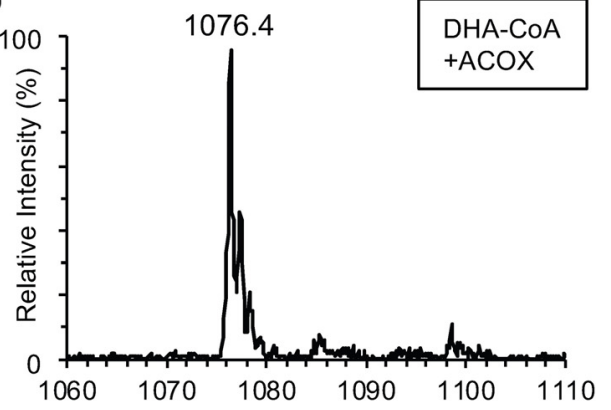

E

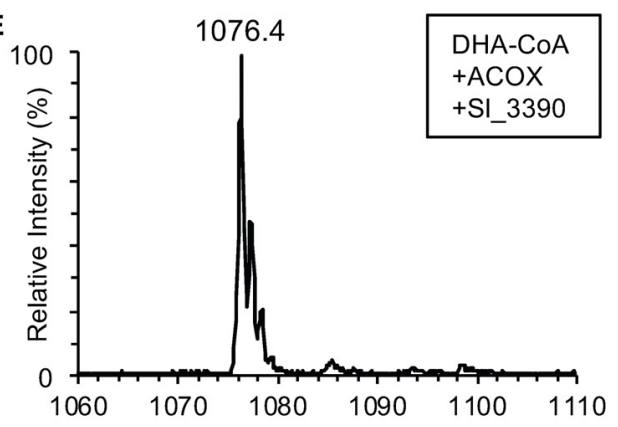

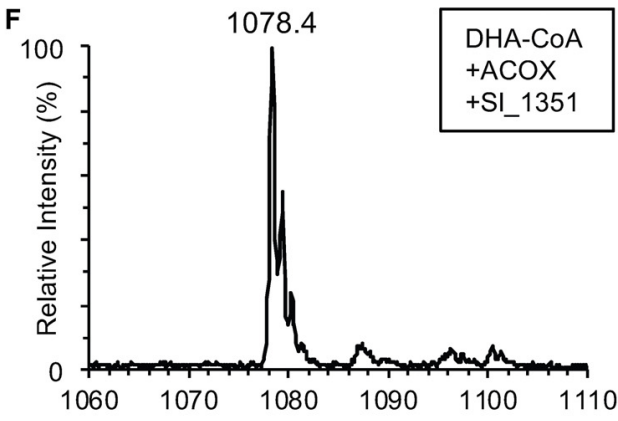

G

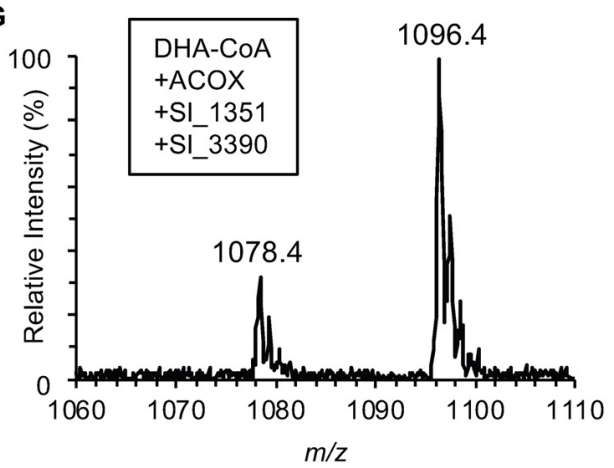

H

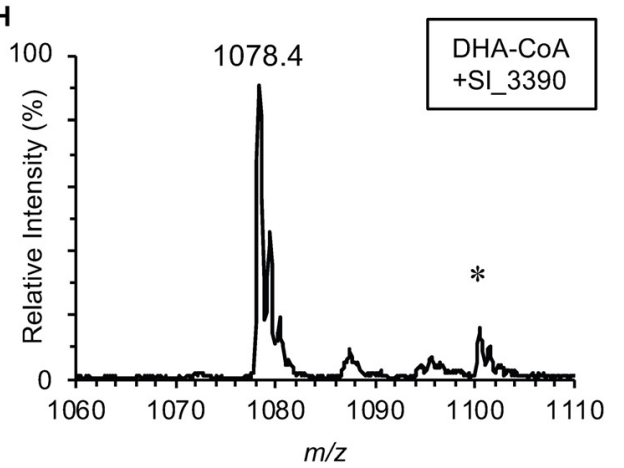

FIGURE 4 | In vitro assay for recombinant SI_1351. SDS-PAGE analysis of the purified recombinant proteins (A; lane 1, SI_3390; lane 2, SI_1351; lane 3, protein standards), a reaction scheme of the $\beta$-oxidation (B; relative mass changes are indicated in the brackets), and ESI-MS analyses of DHA-CoA (C) and its derivatives after incubation with ACOX alone (D), ACOX and SI_3390 (E), ACOX and SI_1351 (F), ACOX, SI_1351 and SI_3390 (G), and SI_3390 alone (H). The m/z values are provided for $[\mathrm{M}+\mathrm{H}]^{+}$ions. The asterisk indicates $[\mathrm{M}+\mathrm{Na}]^{+}$ions with $\mathrm{m} / 2$ larger than the corresponding $[\mathrm{M}+\mathrm{H}]^{+}$ions by 22 .

class of $\omega-3$ long chain PUFAs. Nevertheless, some marine bacteria mainly produce EPA, whereas others mainly DHA (DeLong and Yayanos, 1986; Yazawa, 1996; Nogi et al., 1998; Kawamoto et al., 2009; Hashimoto et al., 2015), suggesting that the bacteria have an obligate requirement for either of these PUFAs. Therefore, we considered whether DHA acts as a substitute for EPA in S. livingstonensis Ac10. In our previous study, supplementation with DHA-containing PLs suppressed growth defects of the $\triangle \mathrm{EPA}$ strain; however, it remained unclear whether DHA itself substituted for EPA, because EPA was formed from supplemented DHA through the conversion pathway (Sato et al., 2008). As conversion was largely suppressed by the disruption of the sl_1351 gene, we reassessed the interchangeability between DHA and EPA using the $\Delta \mathrm{EPA} / \mathrm{sl}_{-} 1351$ strain.
The rationale of the assessment was that if DHA is biologically equivalent to EPA, DHA should restore the cold-sensitive phenotypes of EPA-less mutants, such as growth retardation and filamentous cell formation at $4^{\circ} \mathrm{C}$, as EPA did (Tokunaga et al., 2017) in the absence of S1_1351. As shown in Figure 5A, supplementation with DHA suppressed the growth retardation of the $\Delta \mathrm{EPA} / \mathrm{sl}_{-} 1351$ strain as much as EPA supplementation. DHA supplementation also suppressed filamentation of the cells (Figure 5B). However, the DHA-supplemented cells were longer than the EPA-supplemented cells. At $\mathrm{OD}_{600}$ of approximately 1.0, $90 \%$ of the EPA-supplemented cells reverted to the normal cell length of 2-4 $\mu \mathrm{m}$ (Figure 5B), and the average length was $3.0 \pm 0.8 \mu \mathrm{m}$, which was shorter than that of the nonsupplemented cells at $5.5 \pm 4.3 \mu \mathrm{m}$. On the other hand, only half of the DHA-supplemented cells were 2-4 $\mu \mathrm{m}$ long, and 43\% 

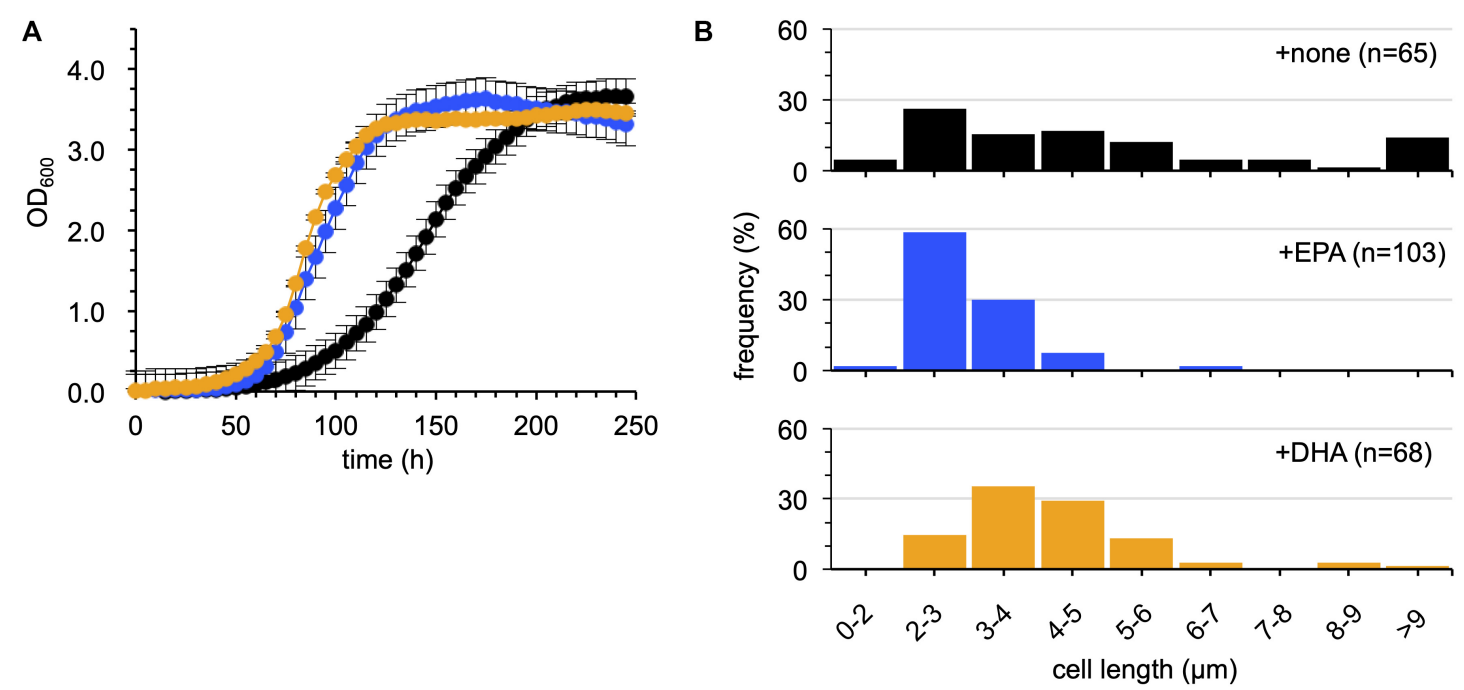

FIGURE 5 | Examination of the effects of EPA and DHA supplementation on the growth of the $\Delta$ EPA/s/_1351 strain. The growth (A) and cell length distribution (B) of non-supplemented (black), EPA-supplemented (blue), and DHA-supplemented (orange) cells are shown. The growth curves were produced according to three independent experiments. The numbers of cells subjected to size measurements are indicated in brackets in each graph.

showed slightly longer lengths of 4-6 $\mu \mathrm{m}$ (the average length, $4.3 \pm 1.7 \mu \mathrm{m})$. Therefore, although DHA is beneficial to the growth of S. livingstonensis Ac10 at cold temperatures, it is not biologically equivalent to EPA.

\section{DISCUSSION}

We previously found that the EPA-producing bacterium S. livingstonensis Ac10 utilized exogenous DHA, added to the culture medium as an acyl group of PL, to produce EPA (Sato et al., 2008). Watanabe et al. (1994) reported the uptake of exogenous DHA by several bacterial species, among which E. coli was found to convert exogenous DHA into EPA. However, the molecular mechanism for the conversion remained to be elucidated. Here, we revealed the conversion mechanisms focusing on the putative $\mathrm{fadH}$ genes. We demonstrated through mutagenesis studies that disruption of the $s l \_1351$ gene markedly suppressed conversion (Figures 1, 2), and through an in vitro enzymatic assay that the recombinant Sl_1351 protein had 2,4-dienoyl-CoA reductase activity toward the 2,4-dienoyl-CoA derivative of DHA (Figure 4). These data strongly suggest that $\mathrm{Sl}_{-} 1351$ is a key enzyme in the conversion of DHA to EPA. However, there remained decreased level of conversion in the $s l$ 1351-disrupted cells. This can be explained by the subtle hydratase activity of FadB toward docosa-2,4,7,10,13,16,19-heptaenoyl-CoA, as Yang et al. (1986) reported that the 2,4-dienoyl-CoA intermediate can be a substrate of FadB and undergo $\beta$-oxidation cycles, although the reactions are thermodynamically unfavorable. Additionally, minor contributions of the other five $\mathrm{FadH}$ homologs are possible.

As Sl_1351 is most homologous to E. coli FadH (56.5\% identical) of the six FadH homologs of S. livingstonensis Ac10, and has both N-terminal catalytic and C-terminal NAD $(\mathrm{P}) \mathrm{H}$ binding domains (Figure 3), it is likely that $\mathrm{Sl} 1351$ is a bona fide $\mathrm{FadH}$ and, thus, the conversion is mediated through a typical $\beta$-oxidation pathway. $\beta$-oxidation enzymes catalyze repeated cycles of reactions to eliminate two carbon units as a form of acetyl-CoA at the end of each cycle and, thus, can sequentially degrade DHA to EPA and further to octadeca3,6,9,12,15-pentaenoic acid and hexadeca-4,7,10,13-tetraenoic acid. However, such PUFAs were undetectable in our ESIMS and GC/MS analyses (data not shown). No PUFAs other than EPA were observed when the cells were supplemented with a synthetic DHA-containing PL (Sato et al., 2008). These observations imply that S. livingstonensis Ac10 preferentially produces EPA from DHA without metabolizing it further and forms EPA-containing PLs, which are important membrane constituents for the bacterium to survive in cold environments (Kawamoto et al., 2009).

Further investigations could determine how the $\beta$-oxidation pathway is ceased after one cycle of reactions to preferentially generate EPA. This regulation is exemplified by the Sprecher pathway used for DHA biosynthesis in mammals. The pathway involves initial production of tetracosa-6,9,12,15,18,21-hexaenoic acid from $\alpha$-linolenic acid via an elongase-desaturase system and the following retroconversion to DHA through a single cycle of $\beta$-oxidation reactions (Voss et al., 1991). Peroxisomal $\beta$-oxidation enzymes are responsible for the chain-shortening step (Su et al., 2001); however, the exact mechanism remains enigmatic. One possible mechanism for the selective conversion of DHA to EPA by S. livingstonensis Ac10 is that the FadE enzyme, which catalyzes the first committed step of the $\beta$-oxidation pathway, is active toward DHA-CoA but not, or to a lesser extent, toward EPA-CoA. If this is the case, the next cycle of the $\beta$-oxidation reaction would not proceed efficiently, and EPA-CoA is preferentially used by $\mathrm{PlsC}$ as the substrate for PL 
formation (Ogawa et al., 2018). S. livingstonensis Ac10 has two FadE homologs that are highly homologous to E. coli FadE, suggesting that at least one of them is active toward DHA-CoA. To examine whether FadE is a key regulator in the conversion of DHA to EPA, we are currently analyzing the in vivo and in vitro characteristics of the FadE homologs.

Our data shown in Figure $\mathbf{5}$ imply that DHA partially substitutes for EPA but is not interchangeable. Given that the abnormal cell elongation of the $\triangle \mathrm{EPA}$ strain is presumably caused by the deficiency in cell division (Kawamoto et al., 2009), the distinct cell length distribution between the EPA- and DHA-supplemented cells suggests that EPA is more favorably exploited for this process. Although the exact function of PUFAs in marine bacteria remains unknown, their hyperflexible polyene hydrocarbon chain is considered to be a key factor, which can render lipid membrane more fluid and flexible and solvate a membrane protein more efficiently than saturated and monounsaturated fatty acids (Antonny et al., 2015; Yoshida et al., 2016). Interestingly, previous studies utilizing a model eukaryotic membrane have suggested that EPA and DHA, in the form of free acid or an acyl chain of PLs, are moderately different from one another in their physicochemical properties such as the degree of conformational changes within membranes and (dis)ordering lipid bilayer, and, thus, potentially have a distinct impact on cellular events (Williams et al., 2012; Leng et al., 2018; Sherratt and Mason, 2018). As cell division involves the dynamic rearrangement of cellular membranes and is achieved by a variety of membrane proteins such as those forming divisome complex, we speculate that it proceeds more efficiently in the presence of membrane PLs containing EPA than those containing DHA due to the difference in their physicochemical properties. Detailed

\section{REFERENCES}

Antonny, B., Vanni, S., Shindou, H., and Ferreira, T. (2015). From zero to six double bonds: phospholipid unsaturation and organelle function. Trends Cell Biol. 25, 427-436. doi: 10.1016/j.tcb.2015.03.004

Binstock, J. F., and Schulz, H. (1981). Fatty acid oxidation complex from Escherichia coli. Methods Enzymol. 71, 403-411. doi: 10.1016/0076-6879(81) 71051-6

Bligh, E. G., and Dyer, W. J. (1959). A rapid method of total lipid extraction and purification. Can. J. Biochem. Physiol. 37, 911-917. doi: 10.1139/o59-099

Cho, H. N., Kasai, W., Kawamoto, J., Esaki, N., and Kurihara, T. (2012). Characterization of 1-acyl-sn-glycerol-3-phosphate acyltransferase from a polyunsaturated fatty acid-producing bacterium, Shewanella livingstonensis Ac10. Trace Nutr. Res. 29, 92-99.

DeLong, E. F., and Yayanos, A. A. (1986). Biochemical function and ecological significance of novel bacterial lipids in deep-sea procaryotes. Appl. Environ. Microbiol. 51, 730-737.

DiRusso, C. C., Black, P. N., and Weimar, J. D. (1999). Molecular inroads into the regulation and metabolism of fatty acids, lessons from bacteria. Prog. Lipid Res. 38, 129-197. doi: 10.1016/s0163-7827(98)00022-8

Dommes, V., and Kunau, W. H. (1984). 2,4-Dienoyl coenzyme A reductases from bovine liver and Escherichia coli, comparison of properties. J. Biol. Chem. 259, 1781-1788.

Garcia, R., Pistorius, D., Stadler, M., and Müller, R. (2011). Fatty acid-related phylogeny of Myxobacteria as an approach to discover polyunsaturated omega3/6 fatty acids. J. Bacteriol. 193, 1930-1942. doi: 10.1128/JB.01091-10 comparative studies regarding EPA and DHA are necessary to clarify their distinctive physiological functions, and the conversion-deficient strain constructed here will contribute to those studies.

\section{DATA AVAILABILITY STATEMENT}

The datasets generated for this study can be found in the DDBJ LC528929, LC528930, LC528931, LC528932, LC528933, LC528934, LC528935.

\section{AUTHOR CONTRIBUTIONS}

TO, JK, and TK designed the study. KH and YY performed the experiments. TO and TK wrote the manuscript. All authors discussed the results.

\section{FUNDING}

This work was supported by JSPS KAKENHI (JP19K15733 to TO and JP18H02127 to TK) and a Grant from the Institute for Fermentation, Osaka (G-2019-2-131 to TO).

\section{SUPPLEMENTARY MATERIAL}

The Supplementary Material for this article can be found online at: https://www.frontiersin.org/articles/10.3389/fmicb. 2020.01104/full\#supplementary-material

Garcia, R., Stadler, M., Gemperlein, K., and Müller, R. (2016). Aetherobacter fasciculatus gen. nov., sp. nov. and Aetherobacter rufus sp. nov., novel myxobacteria with promising biotechnological applications. Int. J. Syst. Evol. Microbiol. 66, 928-938. doi: 10.1099/ijsem.0.000813

Hashimoto, M., Orikasa, Y., Hayashi, H., Watanabe, K., Yoshida, K., and Okuyama, H. (2015). Occurrence of trans monounsaturated and polyunsaturated fatty acids in Colwellia psychrerythraea strain 34H. J. Basic Microbiol. 55, 838-845. doi: 10.1002/jobm.201400815

Hayashi, S., Satoh, Y., Ogasawara, Y., Maruyama, C., Hamano, Y., Ujihara, T., et al. (2019). Control mechanism for cis double-bond formation by polyunsaturated fatty-acid synthases. Angew. Chem. Int. Ed. Engl. 58, 2326-2330. doi: 10.1002/ anie. 201812623

Hubbard, P., Liang, X., Schulz, H., and Kim, J. J. (2003). The crystal structure and reaction mechanism of Escherichia coli 2,4-dienoyl-CoA reductase. J. Biol. Chem. 278, 37553-37560. doi: 10.1074/jbc.M304642200

Ichihara, K., and Fukubayashi, Y. (2010). Preparation of fatty acid methyl esters for gas-liquid chromatography. J. Lipid Res. 51, 635-640. doi: 10.1194/jlr.D00 1065

Ito, T., Gong, C., Kawamoto, J., and Kurihara, T. (2016). Development of a versatile method for targeted gene deletion and insertion by using the pyrF gene in the psychrotrophic bacterium, Shewanella livingstonensis Ac10. J. Biosci. Bioeng. 122, 645-651. doi: 10.1016/j.jbiosc.2016.06.004

Kawamoto, J., Kurihara, T., Yamamoto, K., Nagayasu, M., Tani, Y., Mihara, H., et al. (2009). Eicosapentaenoic acid plays a beneficial role in membrane organization and cell division of a cold-adapted bacterium, Shewanella livingstonensis Ac10. J. Bacteriol. 191, 632-640. doi: 10.1128/JB.00881-08 
Kawamoto, J., Sato, T., Nakasone, K., Kato, C., Mihara, H., Esaki, N., et al. (2011). Favourable effects of eicosapentaenoic acid on the late step of the cell division in a piezophilic bacterium, Shewanella violacea DSS12, at high-hydrostatic pressures. Environ. Microbiol. 13, 2293-2298. doi: 10.1111/j.1462-2920.2011. 02487.x

Leng, X., Kinnun, J., Cavazos, A., Canner, S., Shaikh, S., Feller, S., et al. (2018). All n-3 PUFA are not the same: MD simulations reveal differences in membrane organization for EPA, DHA and DPA. Biochim. Biophys. Acta Biomembr. 1860, 1125-1134. doi: 10.1016/j.bbamem.2018.01.002

Liang, X., Thorpe, C., and Schulz, H. (2000). 2,4-Dienoyl-CoA reductase from Escherichia coli is a novel iron-sulfur flavoprotein that functions in fatty acid B-oxidation. Arch. Biochem. Biophys. 380, 373-379. doi: 10.1006/abbi.2000. 1941

Magnes, C., Sinner, F. M., Regittnig, W., and Pieber, T. R. (2005). LC/MS/MS method for quantitative determination of long-chain fatty acyl-CoAs. Anal. Chem. 77, 2889-2894. doi: 10.1021/ac048314i

Metherel, A. H., and Bazinet, R. P. (2019). Updates to the n-3 polyunsaturated fatty acid biosynthesis pathway: DHA synthesis rates, tetracosahexaenoic acid and (minimal) retroconversion. Prog. Lipid Res. 76:101008. doi: 10.1016/j.plipres. 2019.101008

Nishida, T., Orikasa, Y., Ito, Y., Yu, R., Yamada, A., Watanabe, K., et al. (2006). Escherichia coli engineered to produce eicosapentaenoic acid becomes resistant against oxidative damages. FEBS Lett. 580, 2731-2735. doi: 10.1016/j.febslet. 2006.04.032

Nogi, Y., Masui, N., and Kato, C. (1998). Photobacterium profundum sp. nov., a new, moderately barophilic bacterial species isolated from a deep-sea sediment. Extremophiles 2, 1-7. doi: 10.1007/s00792005 0036

Ogawa, T., Tanaka, A., Kawamoto, J., and Kurihara, T. (2018). Purification and characterization of 1-acyl-sn-glycerol-3-phosphate acyltransferase with a substrate preference for polyunsaturated fatty acyl donors from the eicosapentaenoic acid-producing bacterium Shewanella livingstonensis Ac10. J. Biochem. 164, 33-39. doi: 10.1093/jb/mvy025

Orikasa, Y., Tanaka, M., Sugihara, S., Hori, R., Nishida, T., Ueno, A., et al. (2009). $p f a B$ products determine the molecular species produced in bacterial polyunsaturated fatty acid biosynthesis. FEMS Microbiol. Lett. 295, 170-176. doi: 10.1111/j.1574-6968.2009.01582.x

Sato, S., Kurihara, T., Kawamoto, J., Hosokawa, M., Sato, S., and Esaki, N. (2008). Cold adaptation of eicosapentaenoic acid-less mutant of Shewanella livingstonensis Ac10 involving uptake and remodeling of synthetic phospholipids containing various polyunsaturated fatty acids. Extremophiles 12, 753-761. doi: 10.1007/s00792-008-0182-6

Sherratt, S., and Mason, R. (2018). Eicosapentaenoic acid and docosahexaenoic acid have distinct membrane locations and lipid interactions as determined by X-ray diffraction. Chem. Phys. Lipids 212, 73-79. doi: 10.1016/j.chemphyslip.2018.01. 002

Shulse, C., and Allen, E. (2011). Widespread occurrence of secondary lipid biosynthesis potential in microbial lineages. PLoS One 6:e20146. doi: 10.1371/ journal.pone.0020146

Su, H. M., Moser, A. B., Moser, H. W., and Watkins, P. A. (2001). Peroxisomal straight-chain acyl-CoA oxidase and D-bifunctional protein are essential for the retroconversion step in docosahexaenoic acid synthesis. J. Biol. Chem. 276, 38115-38120. doi: 10.1074/jbc.M106326200

Tokunaga, T., Watanabe, B., Sato, S., Kawamoto, J., and Kurihara, T. (2017). Synthesis and functional assessment of a novel fatty acid probe, $\omega$-ethynyl eicosapentaenoic acid analog, to analyze the in vivo behavior of eicosapentaenoic acid. Bioconjug. Chem. 28, 2077-2085. doi: 10.1021/acs. bioconjchem.7b00235

Toyotake, Y., Cho, H. N., Kawamoto, J., and Kurihara, T. (2018). A novel 1acyl-sn-glycerol-3-phosphate $O$-acyltransferase homolog for the synthesis of membrane phospholipids with a branched-chain fatty acyl group in Shewanella livingstonensis Ac10. Biochem. Biophys. Res. Commun. 500, 704-709. doi: 10. 1016/j.bbrc.2018.04.140

Tu, X., Hubbard, P. A., Kim, J. J., and Schulz, H. (2008). Two distinct proton donors at the active site of Escherichia coli 2,4-dienoyl-CoA reductase are responsible for the formation of different products. Biochemistry 47, 1167-1175. doi: 10.1021/bi701235t

Voss, A., Reinhart, M., Sankarappa, S., and Sprecher, H. (1991). The metabolism of 7,10,13,16,19-docosapentaenoic acid to 4,7,10,13,16,19-docosahexaenoic acid in rat liver is independent of a 4-desaturase. J. Biol. Chem. 266, 19995-20000.

Watanabe, K., Ishikawa, C., Inoue, H., Cenhua, D., Yazawa, K., and Kondo, K. (1994). Incorporation of exogenous docosahexaenoic acid into various bacterial phospholipids. J. Am. Oil Chem. Soc. 71, 325-330.

Williams, J. A., Batten, S. E., Harris, M., Rockett, B. D., Shaikh, S. R., Stillwell, W., et al. (2012). Docosahexaenoic and eicosapentaenoic acids segregate differently between raft and nonraft domains. Biophys. J. 103, 228-237. doi: 10.1016/j.bpj. 2012.06.016

Yang, S. Y., Cuebas, D., and Schulz, H. (1986). 3-Hydroxyacyl-CoA epimerases of rat liver peroxisomes and Escherichia coli function as auxiliary enzymes in the $\beta$-oxidation of polyunsaturated fatty acids. J. Biol. Chem. 261, 12238-12243.

Yazawa, K. (1996). Production of eicosapentaenoic acid from marine bacteria. Lipids 31, S297-S300. doi: 10.1007/bf02637095

Yoshida, K., Hashimoto, M., Hori, R., Adachi, T., Okuyama, H., Orikasa, Y., et al. (2016). Bacterial long-chain polyunsaturated fatty acids: their biosynthetic genes, functions, and practical use. Mar. Drugs 14, 94. doi: 10.3390/ md14050094

You, S. Y., Cosloy, S., and Schulz, H. (1989). Evidence for the essential function of 2,4-dienoyl-coenzyme A reductase in the $\beta$-oxidation of unsaturated fatty acids in vivo. Isolation and characterization of an Escherichia coli mutant with a defective 2,4-dienoyl-coenzyme A reductase. J. Biol. Chem. 264, 16489-16495.

Conflict of Interest: The authors declare that the research was conducted in the absence of any commercial or financial relationships that could be construed as a potential conflict of interest.

Copyright (c) 2020 Ogawa, Hirose, Yusuf, Kawamoto and Kurihara. This is an openaccess article distributed under the terms of the Creative Commons Attribution License (CC BY). The use, distribution or reproduction in other forums is permitted, provided the original author(s) and the copyright owner(s) are credited and that the original publication in this journal is cited, in accordance with accepted academic practice. No use, distribution or reproduction is permitted which does not comply with these terms. 